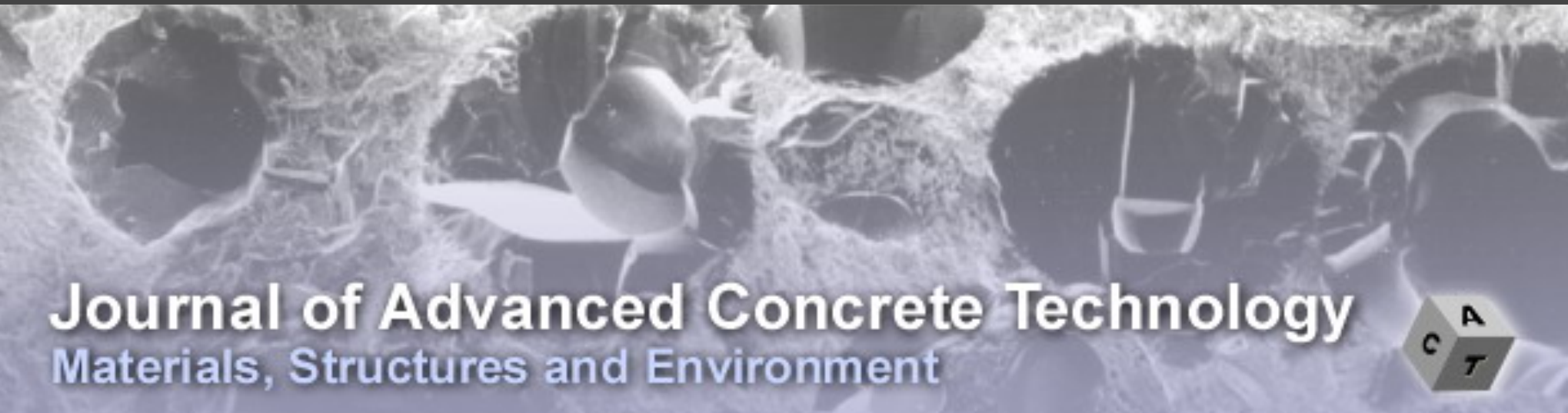

\title{
Effect of Load Pattern in the Generation of Higher Harmonic Amplitude in Concrete Using Nonlinear Ultrasonic Test
}

Jason Maximino Ongpeng, Andres Winston Oreta, Sohichi Hirose

Journal of Advanced Concrete Technology, volume 14 (2016 ), pp. 205-214

\section{Related Papers Click to Download full PDF!}

Nondestructive Evaluation of Damaged Concrete due to Freezing and Thawing by Elastic-Wave Method Masayasu Ohtsu

Journal of Advanced Concrete Technology, volume 3 (2005), pp. 333-341

Ultrasonic Wave Reflection Approach to Evaluation of Fresh Concrete Friction

Yannick Vanhove, Chafika Djelal, Thierry Chartier

Journal of Advanced Concrete Technology, volume 6 (2008), pp. 253-260

Kinematics of Corrosion Damage Monitored by Acoustic Emission Techniques and Based on a Phenomenological Model

Yuma Kawasaki, Tomoe Kobarai, Masayasu Ohtsu

Journal of Advanced Concrete Technology, volume 10 (2012), pp. 160-169

Ultrasonic nondestructive testing of cement grouting quality in corrugated pipes based on impact-echo Qingbang Han, Jin Cheng, Cheng Yin, Honghui Fan, Changping Zhu

Journal of Advanced Concrete Technology, volume 12 (2014), pp. 503-509

\section{Click to Submit your Papers}




\title{
Effect of Load Pattern in the Generation of Higher Harmonic Amplitude in Concrete Using Nonlinear Ultrasonic Test
}

\author{
Jason Maximino C. Ongpeng ${ }^{1}$, Andres Winston C. Oreta ${ }^{2}$ and Sohichi Hirose ${ }^{3}$
}

Received 29 January 2016, accepted 11 May 2016

doi:10.3151/jact.14.205

\begin{abstract}
Non-destructive testing is used in monitoring health of structures. In this paper, nonlinear ultrasonic test was done to analyze the sensitivity of the fundamental and higher harmonics with the internal damage in concrete. Good sensitivity produces significant quantitative changes in the nonlinear spectral amplitude parameter corresponding to the deviation of load.

In actual conditions, structures have different design strength with varying water cement ratio. In addition, it is experiencing loading and unloading. These are considered as major factors that induce effect in the behavior of ultrasonic test result in concrete. Past researches have only considered increasing application of load with single loading pattern. This study aims to investigate the effect of load application pattern in the generation of higher harmonics.

Ordinary concrete specimens of size $150 \mathrm{~mm}$ x $150 \mathrm{~mm}$ x $150 \mathrm{~mm}$ with water-cement ratio WC40 and WC60 were casted and tested using uniaxial compression machine with two load patterns: a.) single loading pattern from $0 \%$ to $100 \%$ and, b.) multiple loading/unloading pattern. Ultrasonic test with pitch-catch configuration was conducted at each loading state to record the fundamental and higher harmonic generation in the concrete.

The experimental results showed that there was a good sensitivity for $3^{\text {rd }}$ harmonic amplitude parameter $\mathrm{D}_{\mathrm{A}}$ when it was subjected to single loading pattern for both WC40 and WC60. On the other hand, good sensitivity was only observed for $2^{\text {nd }}$ harmonic amplitude parameter $\mathrm{D}_{\mathrm{A}}$ when it was subjected to multiple loading/unloading pattern for both WC40 and WC60. This showed that the $2^{\text {nd }}$ harmonic amplitude was consistent and sensitive when repeated load was applied for low and high water cement ratio.
\end{abstract}

\section{Introduction}

\subsection{Linear and nonlinear ultrasonic test in con- crete}

Concrete has been predominant in structures. In its early life, concrete usually develops micro cracks between the aggregates and bonding agent. With the application of loads, new micro cracks occur, and early cracks grow further to larger cracks (Stauffer et al. 2005). In addition, seismic movement of the structure induce propagation of micro cracks until failure occurs. Thus, concrete inspection at micro level should be done to monitor its serviceability throughout the structure's lifespan.

Non-destructive testing is a widely used method to examine the structural integrity of the concrete structure at micro level. Unlike other materials, concrete is complex. There are a lot of linear ultrasonic testing procedures in concrete from the past. An example of linear ultrasonic test is Ultrasonic Pulse Velocity wherein time of wave traveling a particular distance through the thickness of the concrete are the parameters used to derive the velocity. It is worth noting that in this test, it is

\footnotetext{
${ }^{1}$ Assistant Professor, De La Salle University, Manila, Philippines. *Corresponding author, E-mail: jason.ongpeng@dlsu.edu.ph

${ }^{2}$ Professor, De La Salle University, Manila, Philippines

${ }^{3}$ Professor, Tokyo Institute of Technology, Tokyo, Japan.
}

not essential to measure the wave amplitude.

Combination of linear ultrasonic test using ultrasonic pulse velocity (UPV) and rebound hammer was introduced to test on site strength of concrete (Breysse 2012). Another combination of ultrasonic pulse velocity (UPV) and ultrasonic pulse amplitude (UPA) was formulated to predict the compressive strength of concrete (Liang and Wu 2002). With any combination to improve the prediction of strength of concrete, ultrasonic pulse velocity (UPV) is still limited due to its insensitivity to the changes in load (Daponte et al. 1995). Other tests like air-coupled impact echo (IE), infrared (IR), and sounding (chain drag) method were introduced to the nondestructive method in concrete (Oh et al. 2013). It was emphasized that cracks of size greater than $100 \mathrm{~mm}$ were the ones detected by longitudinal ultrasonic pulses (Komlos et al. 1996). The study further claimed that cracks were practically undetectable especially if it is filled up with fluids.

Another method of non-destructive test is nonlinear ultrasonic test. This provides more promising results due to its sensitivity in damage and crack detection. This test considers harmonic generation which is a phenomenon resulting from interaction between concrete and ultrasonic wave (Zheng et al. 1999). Nonlinear ultrasonic waves proved to be sensitively interacting with contact-type defects (Yim et al. 2012). This includes the opening and closing of cracks formed when loading and unloading occurs. Ultrasonic waves passing thru dam- 
aged concrete interacts with micro cracks that result to generation of higher harmonics. In particular, harmonic ratio generated from damaged concrete is sensitive to micro structural changes and micro-cracking in the interfacial transition zone (Shah and Ribakov 2009). Variation of concrete mixture also influences the generation of higher harmonics. Increase in water-cement ratio in concrete proved to be increasing with the nonlinear parameter. In previous studies, small crack opening displacement produces large 2nd higher harmonics (Hirose and Achenbach 1993). From previous experiments, third harmonic ratio is more sensitive compared to the $2^{\text {nd }}$ harmonic ratio in a single loading branch (Shah et al. 2009). Defect detection using $3^{\text {rd }}$ harmonic generation is also sensitive to damage (Shah and Ribakov 2008). It is also suggested that the amplifier be triggered at high power level in experiments to produce better sensitivity in the higher harmonics generation (Shah et al. 2013).

A theory that can be introduced to complex material like concrete is called Nonlinear Mesoscopic Elastic (NME) materials. This results to several important properties such as shift of resonance frequency, generation of higher harmonics, and the phenomenon known as slow dynamics (Johnson and Sutin 2005). These effects are called non-classical non-linear where there are softer regions inside a hard material. Some examples of failure are micro cracks in soft bonding regions between grains. In particular for a concrete specimen, the presence of cement paste which served as a soft bonding region binds with aggregates of different sizes together which served as a hard material in the matrix. If external forces are applied to a concrete specimen, some weak joints may be broken since it is relatively weaker than the coarse aggregates. For weak joints, nonlinear behavior sets in at an earlier stage. For strong joints, it behaves linearly and then nonlinearly at the latter part. This paper aims to consider weak and strong joints of the cement paste between aggregates with the presence of different water cement ratio used. The low water cement ratio of 0.40 considered strong joints with presence of less air voids, while the high water cement ratio of 0.60 considered weak joints with presence of more air voids.

\subsection{Nonlinear ultrasonic methods and parame- ters}

Previous works on nonlinear methods include Scaling Subtraction Method (SSM) and Spectral Analysis (Antonaci et al. 2010). In this paper, spectral analysis was used to determine the sensitivity of the nonlinear ultrasonic parameters to the damage progression in the concrete cubes. This is to filter time domain waveforms to frequency domain waveforms to analyze the frequency content of the elastic response of the medium and to evaluate the amplitude of the newly generated higher order frequencies and their dependence on the amplitude of the fundamental frequency of excitation. De- rived from harmonic generation are harmonic ratios. These are nonlinear ultrasonic properties that refer to the ratio between the higher harmonic amplitude and the fundamental amplitude. Harmonic amplitudes are peak amplitudes that can be measured, during the harmonic generation, other than the fundamental one. Harmonic ratio is another parameter utilized to measure nonlinear response of concrete to ultrasonic testing and correlated to development of internal damage in concrete.

The calculation of harmonic ratio is derived using Taylor series expansion to solve for the solution of the nonlinear partial differential equation of motion shown in Eq. 1.

$$
\frac{\partial^{2} U}{\partial t^{2}}=c_{o}^{2}\left[1+\frac{C^{\prime \prime}}{C^{\prime}} \frac{\partial u}{\partial x}+\ldots\right] \frac{\partial^{2} U}{\partial x^{2}}
$$

where $U$ is the particle displacement with respect to time, $t ; x$ is the propagation distance; $C$ " and $C$ " " are the $2^{\text {nd }}$ and $3^{\text {rd }}$ order elastic constants and $c_{\mathrm{o}}$ is the velocity of the wave.

Since harmonics are generated when fundamental ultrasonic signals encounter damage, solutions are offered to relate the fundamental amplitude with the generated amplitudes of the higher harmonics in Eqs. 2 and 3.

$$
\begin{aligned}
& U_{2}=-\frac{\Gamma_{2}}{4} k^{2} U_{o}{ }^{2} x \\
& U_{3}=-\frac{\Gamma_{2}^{2}}{8} k^{4} U_{o}^{3} x^{2}
\end{aligned}
$$

where $U_{2}$ and $U_{3}$ are now the solution for Eq. $1 . \Gamma_{2}$ is $C$ "' $/ 2 C^{\prime \prime}$. The parameters $U_{2}$ and $U_{3}$ are now the harmonic amplitudes as related to the fundamental amplitude, $U_{\mathrm{o}}$. To take into account the attenuation (wave loss of amplitude) of fundamental signal when wave encounters damage and the generation of higher harmonics, the calculation for harmonic ratios are presented in Eqs. 4 and 5.

$$
\begin{aligned}
& \frac{A_{2}}{A_{1}{ }^{2}}=-\frac{\Gamma_{2}}{4} k^{2} x \\
& \frac{A_{3}}{A_{1}^{3}}=-\frac{\Gamma_{2}}{8} k^{4} x^{2}
\end{aligned}
$$

where $A_{1}, A_{2}$, and $A_{3}$ are the fundamental, second, and third harmonic amplitudes taken to be approximately equal to the fundamental, second, and third harmonic displacements $\left(U_{1}, U_{2}\right.$ and $\left.U_{3}\right) ; k$ is the wave number.

Harmonic generation will not occur without attenuation as mentioned above. Attenuation is the reduction in intensity in any kind of flux during its travel through a medium. This is an ultrasonic wave response when it propagates through a material in which its energy gets reduced as wave scatters due to the presence of microscopic interfaces. Moreover, internal friction contributes to the attenuation. In other words, attenuation is an energy loss within the material experienced by the sound 
Table 1 Design mix of concrete.

\begin{tabular}{|c|c|c|c|c|c|c|c|}
\hline \multirow{2}{*}{ TYPE } & \multirow{2}{*}{ Maximum Size of Gravel (mm) } & \multirow{2}{*}{ W/C (\%) } & \multicolumn{4}{|c|}{ Unit Quantity $\left(\mathrm{kg} / \mathrm{m}^{3}\right)$} \\
\cline { 4 - 8 } & & & Water & Cement & Sand & Gravel & Water-reducing agent \\
\hline WC40 & \multirow{2}{*}{20} & 40 & 169 & 422 & 702 & 1039 & 0.84 \\
\cline { 4 - 8 } WC60 & & 60 & 175 & 291 & 812 & 1021 & 0.58 \\
\hline
\end{tabular}

wave as it travels through the medium.

There were few studies in relating higher harmonic amplitudes with the contacting surface of materials. Recent work showed that the second harmonic generation at the contacting surface of two aluminum blocks was experimentally observed (Murase et al. 2014). Only few researches studied nonlinear ultrasonic test in concrete under loading and unloading conditions with strong and weak joints. In this paper, the effect of loading and unloading in concrete was investigated to define the distinction between initial and latter loading branches of degradation. Furthermore, the harmonic ratio $\left(A_{2} / A_{1}{ }^{2}\right.$ and $\left.A_{3} / A_{1}{ }^{3}\right)$ from equations 4 and 5 were not used in this paper, rather the individual harmonic generations $A_{1}, A_{2}$, and $A_{3}$ were used as a parameter to define the sensitivity with different loading pattern and water-cement ratio.

\section{Experimental procedures}

\subsection{Specimens}

A total of 22 cubic specimens of size $150 \mathrm{~mm} \times 150 \mathrm{~mm}$ $\mathrm{x} 150 \mathrm{~mm}$ were casted. The water-cement ratio was varied as WC40 and WC60. The concrete contained maximum size of aggregates of $20 \mathrm{~mm}$ and sand-total aggregate ratio of $45 \%$. The mixture is shown in Table 1 .

\subsection{Experimental setup}

After curing, all the specimens were at their mature age, a universal testing machine was used to subject each specimen to compression test. There were two experimental set-up established in this paper. Fourteen concrete cubes were tested in a single loading pattern from $0 \%$ to $100 \%$. The remaining eight concrete cubes were tested using multiple loading/unloading pattern as shown in Fig. 1. In this figure, there were four loading branches considered. These had percent load of $0 \%$ to $20 \%, 0 \%$ to $40 \%, 0 \%$ to $60 \%$, and $0 \%$ to $100 \%$, respectively.

The experimental setup is shown in Fig. 2. Specimens were tested by compressive loading. After every loading or unloading, nonlinear ultrasonic testing was used to assess the sample.

The nonlinear ultrasonic test setup is composed of a tone-burst pulser, a high gain broadband receiver, two transducers, and an oscilloscope. The tone-burst pulser supplied voltage amounting up to $1800 \mathrm{~V}$ with 10 cycles of $100 \mathrm{kHz}$ Sine Wave. The pulser-receiver unit has built in modern facilities such as low pass filter set at $3 \mathrm{MHz}$ and high pass filter set at $50 \mathrm{kHz}$, and input impedance of $50 \Omega$.

Transducers, connected to the tone-burst pulser and broadband receivers, were carefully aligned, centered and bonded to the concrete specimen using couplant. A transducer with $100 \mathrm{kHz}$ generating frequency capacity was used to transmit the signal from one face of the

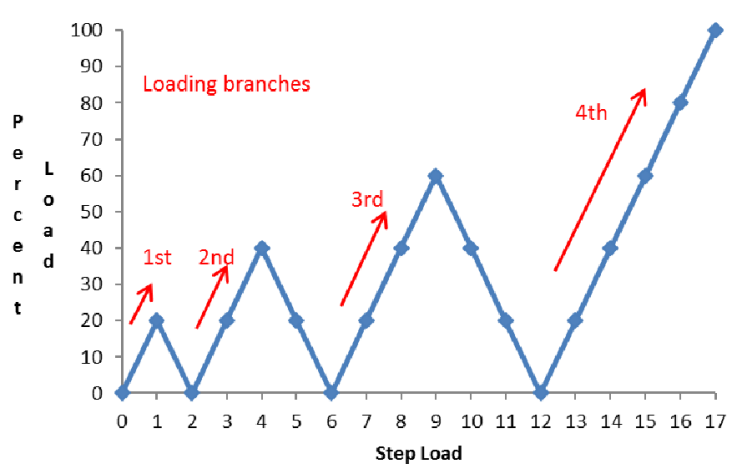

Fig. 1 Multiple loading/unloading pattern.

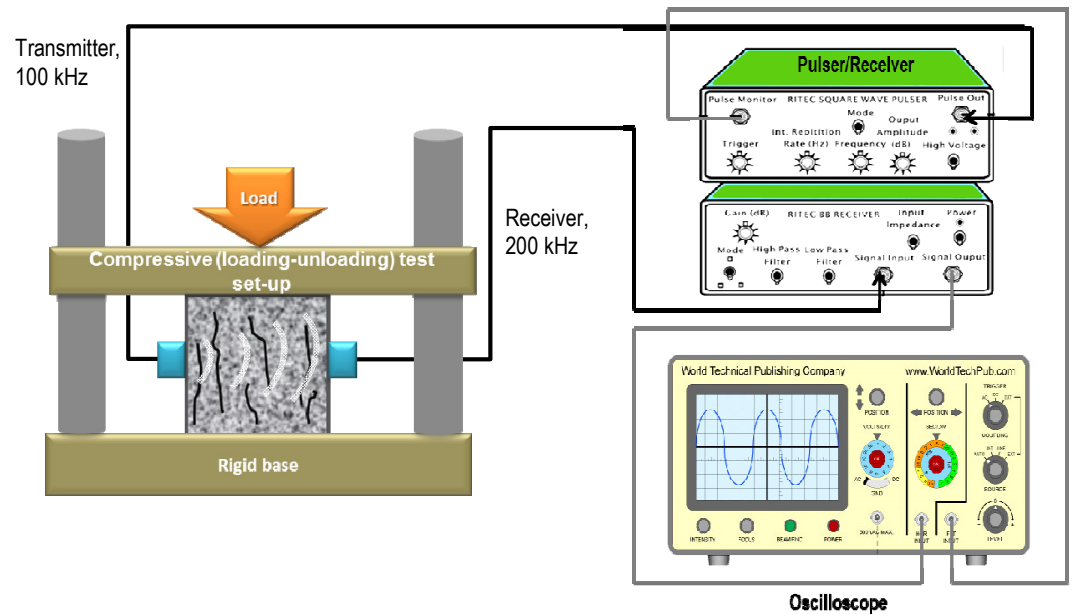

Fig. 2 Compressive testing procedure by loading and unloading setup using nonlinear ultrasonic testing. 
specimen. These ultrasonic signals/waves propagate through the damaged concrete. Receiving transducer with $200 \mathrm{kHz}$ frequency was placed at the opposite face of the specimen that detected the signal passing through the medium. Ultrasonic signals being transmitted and received were translated through the oscilloscope at each step load. All tested specimens were transmitted with signals at $100 \mathrm{kHz}$ fundamental frequency.

\subsection{Higher harmonic generation}

Higher harmonic waves occur in concrete due to contact of crack interfaces called Contact Acoustic Nonlinearity (CAN) (Solodov et al. 2002). This is done through opening and closing of cracks and/or frictional forces acting on the interfaces between cement paste and the aggregates. The higher harmonics generated depends on the behavior of the cracks forming inside when corresponding compressive load is applied (Solodov and Chin 1993).

The transmitted and received frequency domain pass- ing through concrete is shown in Fig. 3. The generation of higher harmonics prove that the concrete material is nonlinear with the presence of its ingredients and microcracks.

The results obtained at the receiving transducer were plotted in a time-domain waveform, and then converted to frequency-domain using Fast Fourier Transform (FFT). Afterwards, the amplitudes of fundamental and higher harmonics in frequency-domain were measured.

\section{Discussion of results}

\subsection{Harmonic generation applied to a single loading pattern}

After obtaining the time-domain waveform from the receiving transducers in the experimental set-up of single loading pattern, it was then converted to frequency domain. Shown in Fig. 4 is the frequency spectrum for specimen \#1 with water cement ratio of 0.60 .

It can be observed that there are formations of higher
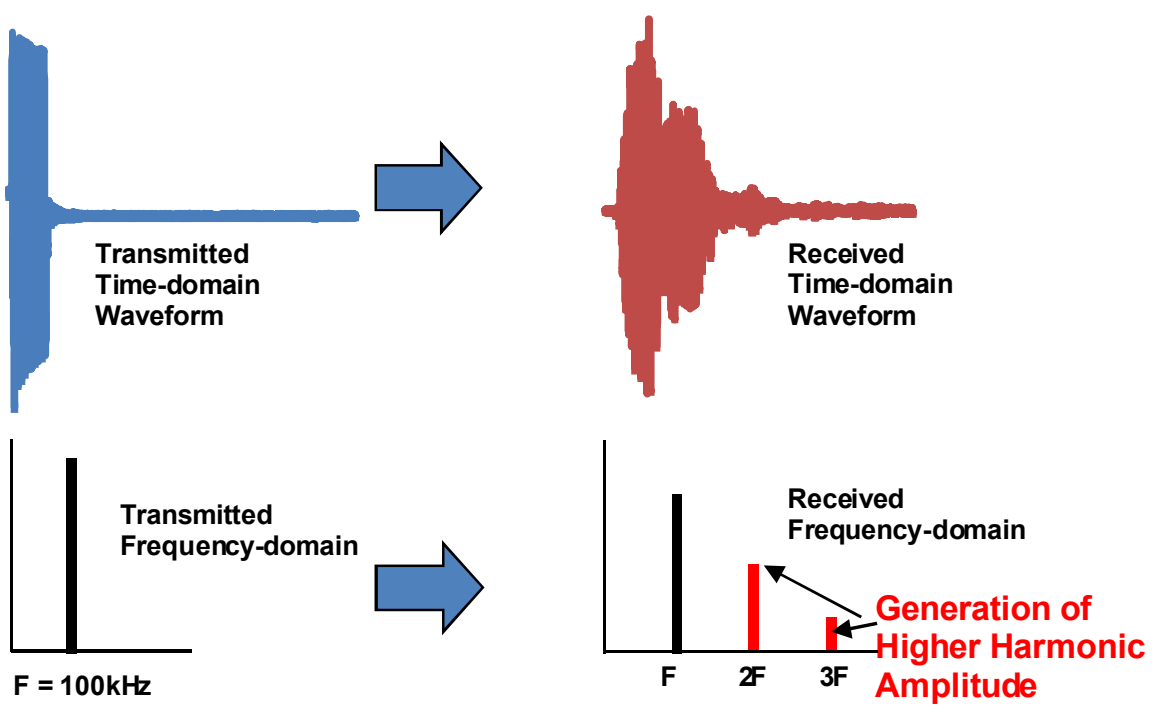

Fig. 3 Transmitted and received wave in time and frequency domain through concrete.

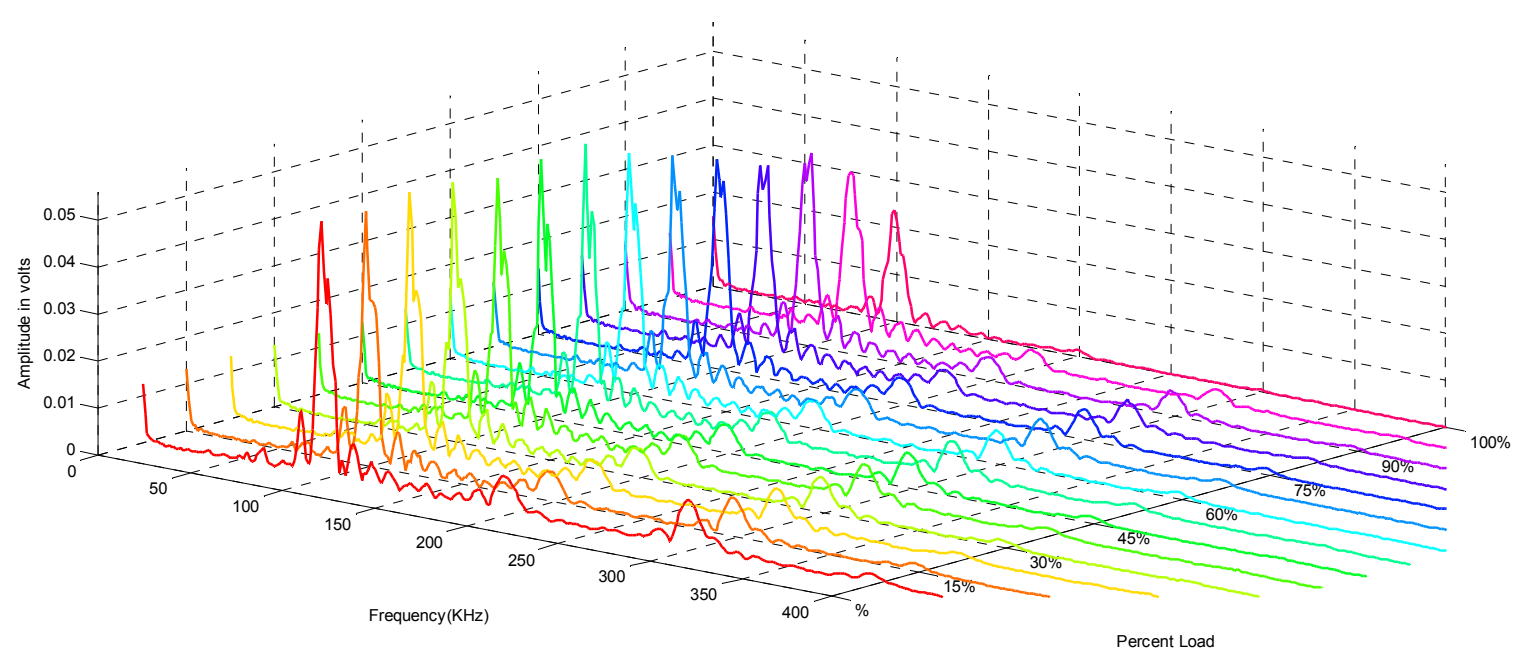

Fig. 4 Frequency spectrum of WC 60 specimen \#1. 
harmonics at $200 \mathrm{kHz}$ and $300 \mathrm{kHz}$. The presence of this higher harmonics, when load is increased, signifies the presence of nonlinear interactions in the concrete.

\subsection{Sensitivity of fundamental and higher har- monic amplitude applied to a single loading pattern}

From a study by Daponte et al., amplitude sensitivity analysis was introduced as shown in Eq. 6.

$$
D_{\mathrm{A}}=1-\mathrm{f}(x) / \mathrm{f} \max
$$

where $D_{\mathrm{A}}$ is the sensitivity of amplitude, $\mathrm{f}(x)$ is the amplitude at current load $x$, fmax is the peak amplitude in the given frequency and loading branch. Sensitivity is the measurement where there is a significant quantitative change with respect to the load applied.

In this paper, the values of $D_{\mathrm{A}}$ were plotted against percent load. Linear regression was used to show the best fit line that represented the $D_{\mathrm{A}}$ equation as a function of percent load. Shown in Fig. 5 is the sensitivity parameter $D_{\mathrm{A}}$ as a function of load. The definition of good sensitivity used in this paper was marked as 0.80 at $100 \%$ load or having a best fit line that had $D_{\mathrm{A}}>$ $0.008 x$, where $x$ is the load percent.

Shown in Fig. $\mathbf{6 a}$ and $\mathbf{6 b}$ are the average values of sensitivity $D_{\mathrm{A}}$ for each harmonic generation for WC 40 and 60 done in the experiment containing single loading pattern. For WC40, $2^{\text {nd }}$ and $3^{\text {rd }}$ harmonic amplitude be-

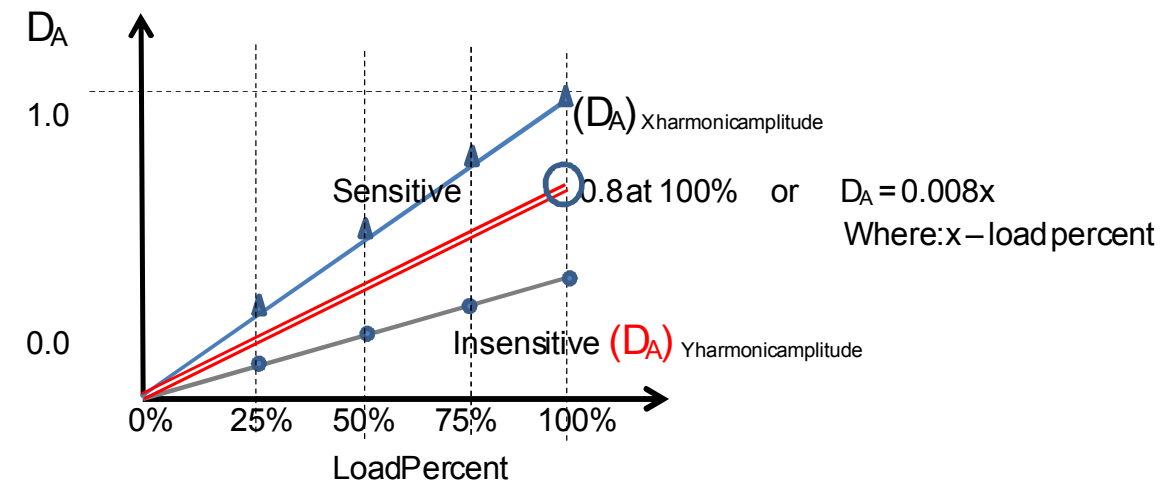

Fig. 5 Sensitivity parameter $D_{A}$ as a function of load.

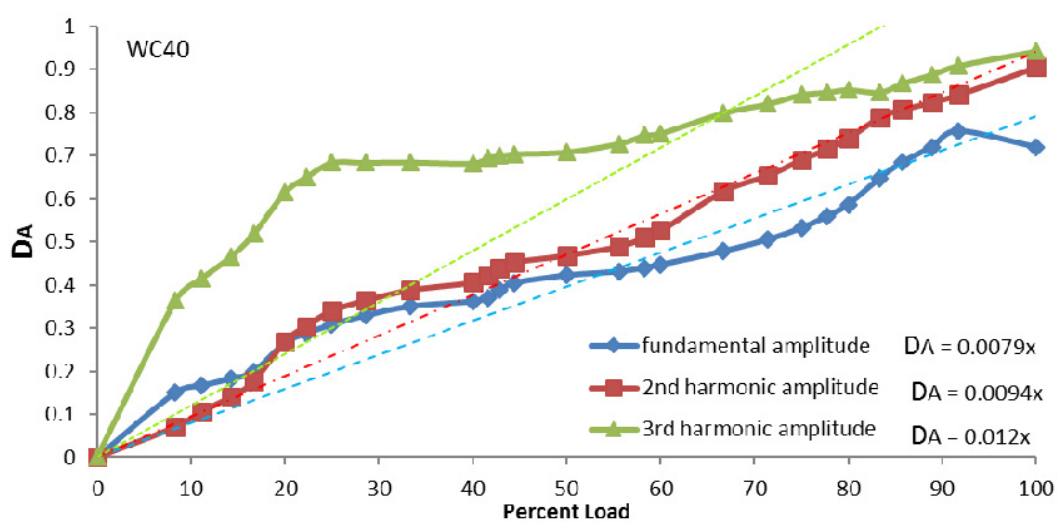

Fig. 6a Sensitivity parameter $D_{A}$ for all harmonic amplitude with water-cement ratio of 0.40 .

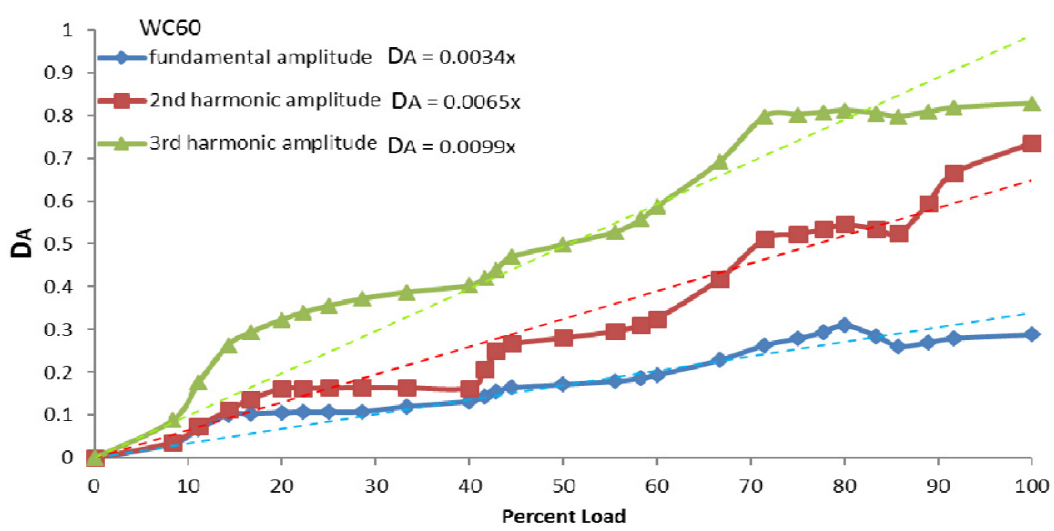

Fig. $6 b$ Sensitivity parameter $D_{A}$ for all harmonic amplitude with water-cement ratio of 0.60 . 
haved with good sensitivity having its best fit line $D_{\mathrm{A}}>$ $0.008 x$. On the other hand, the fundamental and $2^{\text {nd }}$ harmonic amplitude was less sensitive compared to the $3^{\text {rd }}$ harmonic amplitude for concrete with WC60. In summary, the $3^{\text {rd }}$ harmonic amplitude gave good sensitivity to monitor damage in concrete with strong and weak joints represented by WC40 and WC60 respectively.

\subsection{Sensitivity of fundamental and higher har- monic amplitude applied to multiple load- ing/unloading pattern}

Shown in Figs. $\mathbf{7 a}, \mathbf{7 b}$, and $\mathbf{7 c}$ are the sensitivity parameter $D_{\mathrm{A}}$ for the fundamental, $2^{\text {nd }}$ and $3^{\text {rd }}$ harmonic amplitude from the $1^{\text {st }}$ loading branch to the $4^{\text {th }}$ loading branch of concrete with WC40. The best fit line's slope for the fundamental amplitude $1^{\text {st }}$ to $4^{\text {th }}$ loading branch diminishes significantly. As a result, there is less sensitivity at the last loading branch. Similarly, there was a diminishing slope for the $2^{\text {nd }}$ and $3^{\text {rd }}$ harmonics $D_{\mathrm{A}}$ as loading branch increased. The reduction of the slope for both $2^{\text {nd }}$ and $3^{\text {rd }}$ harmonic amplitude was small that kept the best fit line's slope more than 0.80 . This was consistent with the single loading branch were $2^{\text {nd }}$ and $3^{\text {rd }}$ harmonic amplitude was sensitive for strong joints represented by WC40.

In Figs. 8a, 8b, and 8c, it shows the sensitivity parameter $D_{\mathrm{A}}$ for the fundamental, $2^{\text {nd }}$ and $3^{\text {rd }}$ harmonic amplitude from the $1^{\text {st }}$ loading branch to the $4^{\text {th }}$ loading branch of concrete with WC60. The best fit line's slope

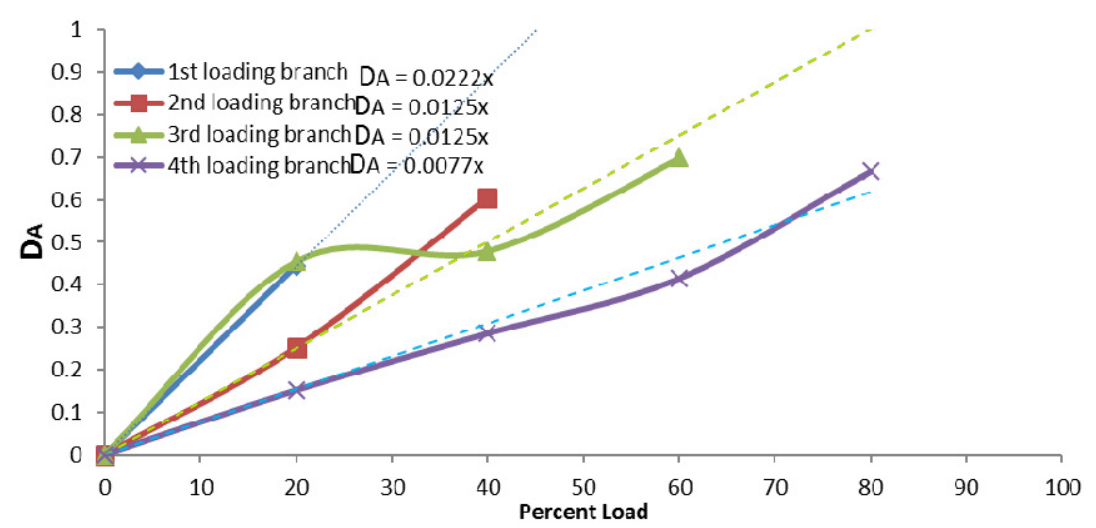

Fig. 7 a Sensitivity parameter $D_{A}$ for fundamental amplitude with water-cement ratio of 0.40 .

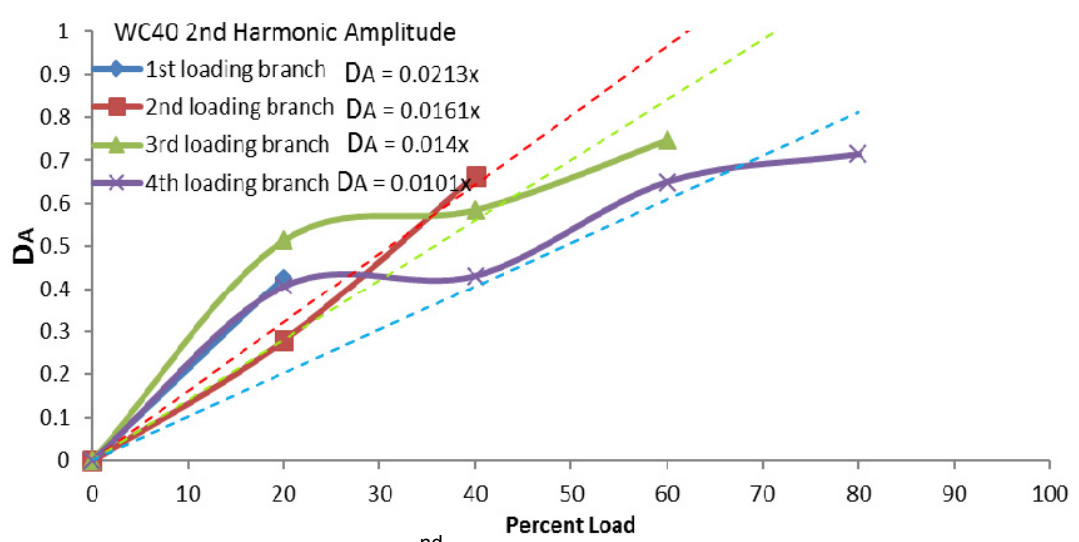

Fig. $7 \mathrm{~b}$ Sensitivity parameter $D_{\mathrm{A}}$ for $2^{\text {nd }}$ harmonic amplitude with water-cement ratio of 0.40 .

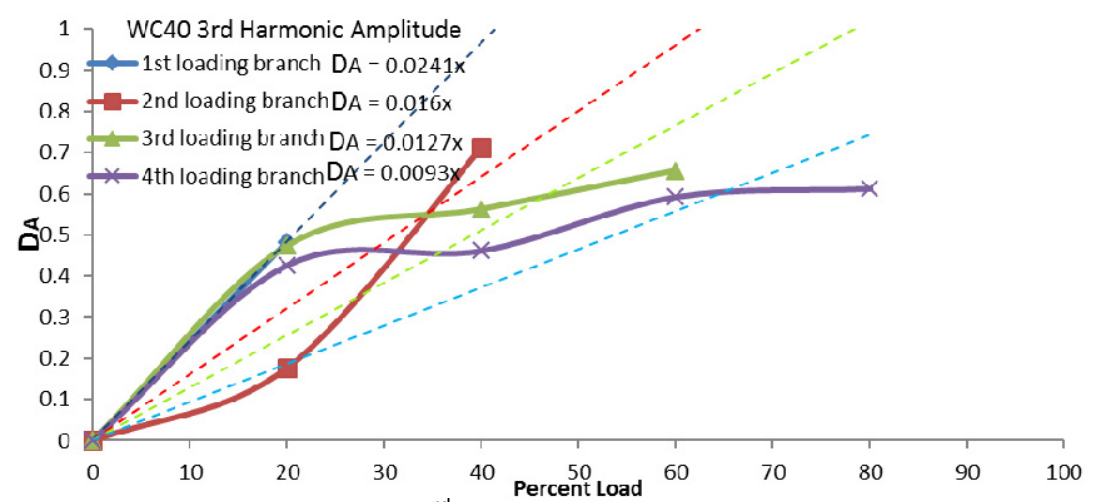

Fig. $7 c$ Sensitivity parameter $D_{A}$ for $3^{\text {rd }}$ harmonic amplitude with water-cement ratio of 0.40 . 
for the fundamental, $2^{\text {nd }}$ and $3^{\text {rd }}$ harmonic amplitude from the $1^{\text {st }}$ to $4^{\text {th }}$ loading branch diminishes. Unlike the concrete with $\mathrm{WC} 40$, the $3^{\text {rd }}$ harmonic amplitude for WC60 had significant reduction of slope from 0.024 to 0.0047 . This shows that for weak joints with WC60 concrete mixture, the sensitivity of the $3^{\text {rd }}$ harmonic amplitude reduces when repeated load is applied. The $2^{\text {nd }}$ harmonic amplitude however remains to be consistent as the parameter sensitive to the load changes with a slope of 0.0091 at the last loading branch.

\subsection{Normalized higher harmonic ratio applied to a single loading pattern}

Higher harmonic amplitude in Fast Fourier Transform has no basis for comparison from the undamaged state of concrete. Normalized higher harmonic ratio is introduced in this paper to set a baseline value. These values are calculated in order to ascertain the effect of loading pattern to the ultrasonic wave propagation in the damaged concrete.

From the frequency spectrum, normalization of amplitude was computed using Equation7

$$
X=20 \log \left(A_{1} / A_{0}\right)
$$

where $X$ is normalized higher harmonic ratio in $\mathrm{dB}, A_{1}$ is amplitudes in volts at a particular step load and $A_{\mathrm{o}}$ is reference amplitude in volts (undamaged state).

The average normalized higher harmonic ratio for the experimental set-up of single loading pattern from a load of $0 \%$ to $100 \%$ is shown in Fig. 9a and $\mathbf{9 b}$. Clear

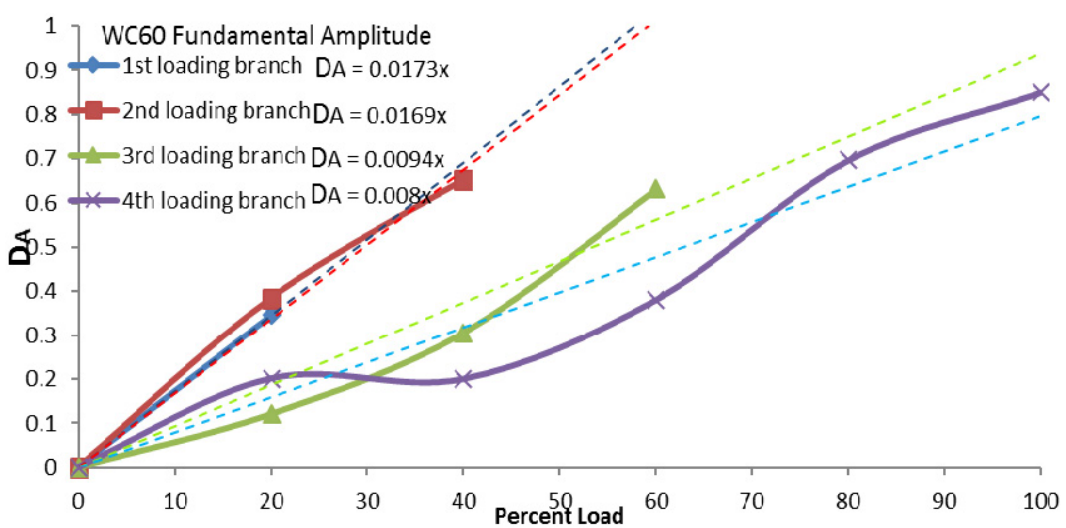

Fig. 8a Sensitivity parameter $D_{A}$ for fundamental amplitude with water-cement ratio of 0.60 .

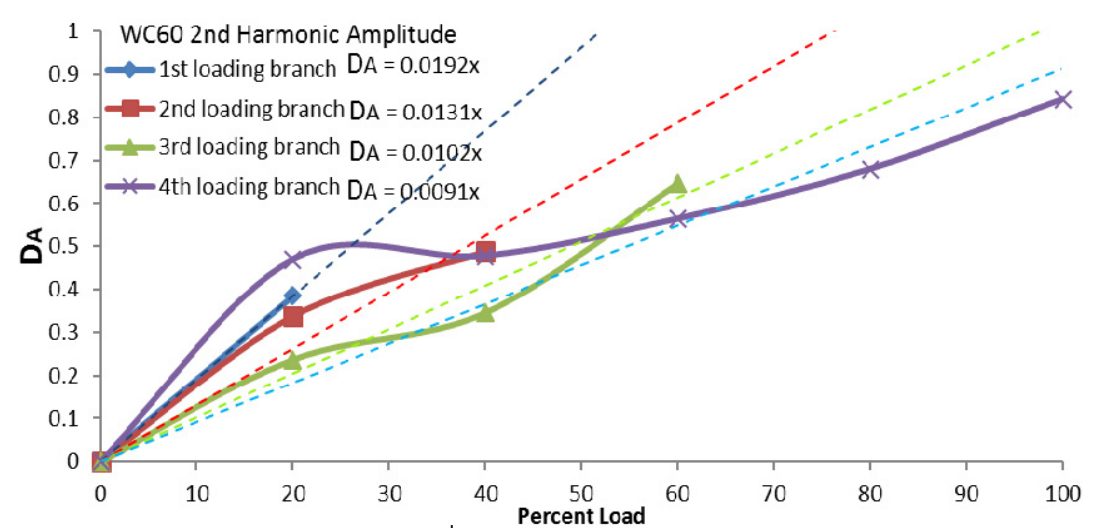

Fig. 8b Sensitivity parameter $D_{\mathrm{A}}$ for $2^{\text {nd }}$ harmonic amplitude with water-cement ratio of 0.60 .

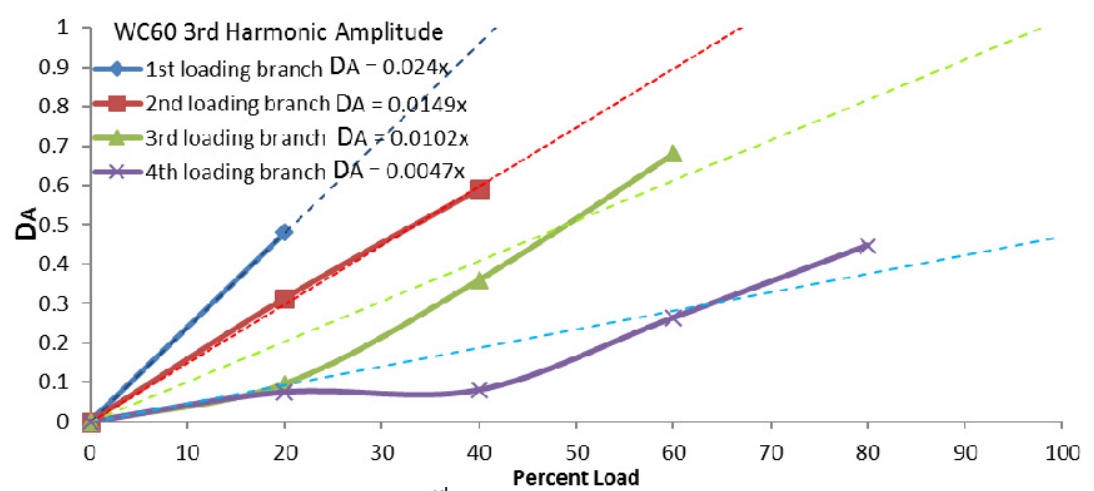

Fig. $8 \mathrm{c}$ Sensitivity parameter $\mathrm{D}_{\mathrm{A}}$ for $3^{\text {rd }}$ harmonic amplitude with water-cement ratio of 0.60 . 


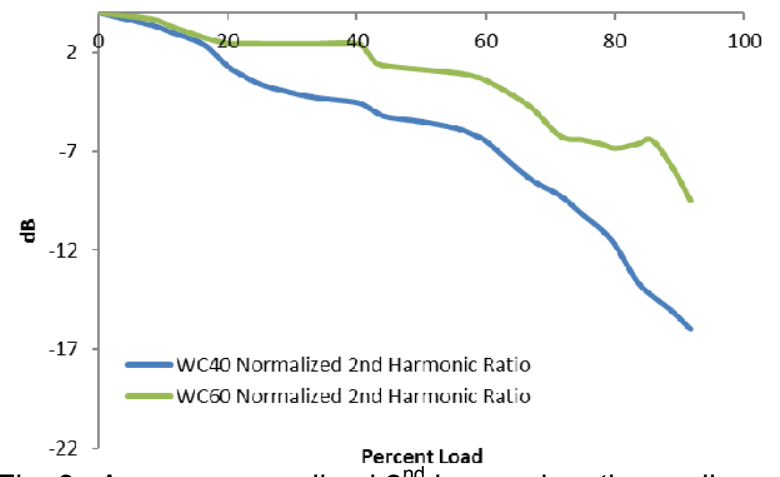

Fig. 9a Average normalized $2^{\text {nd }}$ harmonic ratio amplitude.

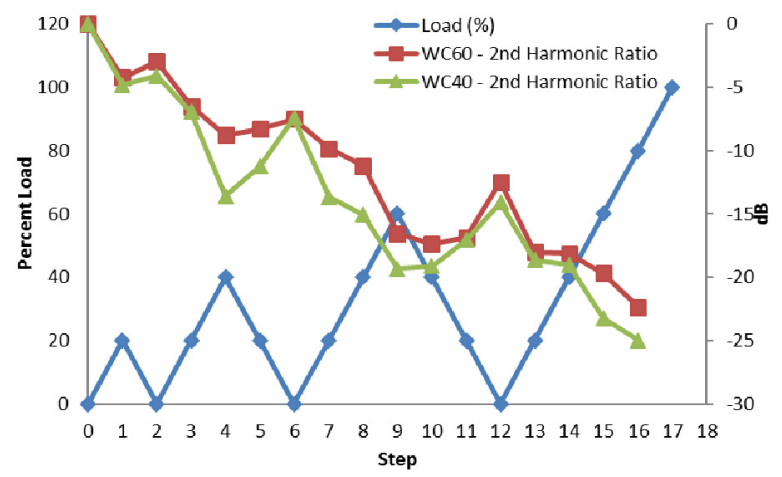

Fig. 10a Average 2nd harmonic amplitude with loading/unloading pattern.

trend showed that it is decreasing with an increasing load. The maximum difference for the average normalized $2^{\text {nd }}$ harmonic ratio of undamaged state at $0 \%$ and the damaged state at $100 \%$ was $-15.96 \mathrm{~dB}$ for $\mathrm{WC} 40$ and $-9.50 \mathrm{~dB}$ for WC60. The difference for the average normalized $3^{\text {rd }}$ harmonic ratio of undamaged state at $0 \%$ and the damaged state at $100 \%$ were $-20.79 \mathrm{~dB}$ for WC40 and $-14.79 \mathrm{~dB}$ for WC60. It showed that the normalized $3^{\text {rd }}$ harmonic ratio is sensitive to damage since it contained higher numerical value for better assessment of internal damage. This behavior was also observed from the previous sensitivity measurement $D_{\mathrm{A}}$ that the $3^{\text {rd }}$ harmonic amplitude was highly sensitive to changes in load.

\subsection{Normalized higher harmonic ratio applied to a loading/unloading pattern}

The time-domain waveforms from the receiving transducers for the loading/unloading branch were recorded. It was then converted to frequency domain. Shown in Fig. 10a and 10b are the normalized harmonic ratios for WC40 and W60. For WC40, all higher harmonic amplitudes were sensitive to loading and unloading pattern. This was evident with the significant variation with respect to changes in load. For WC60, the $2^{\text {nd }}$ harmonic amplitude was sensitive to the loading and unloading pattern compared to WC40.

The normalized $3^{\text {rd }}$ harmonic ratio of WC60 was insensitive due to weak regions represented by weak cement paste or more voids in a heterogeneous material

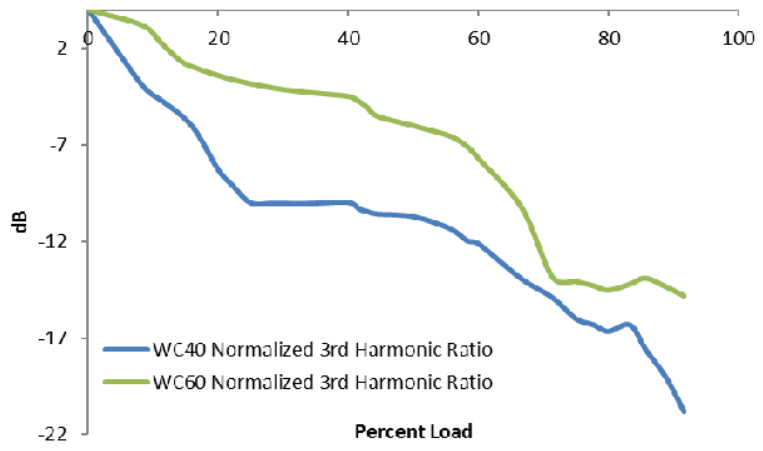

Fig. 9b Average normalized 3rd harmonic ratio amplitude.

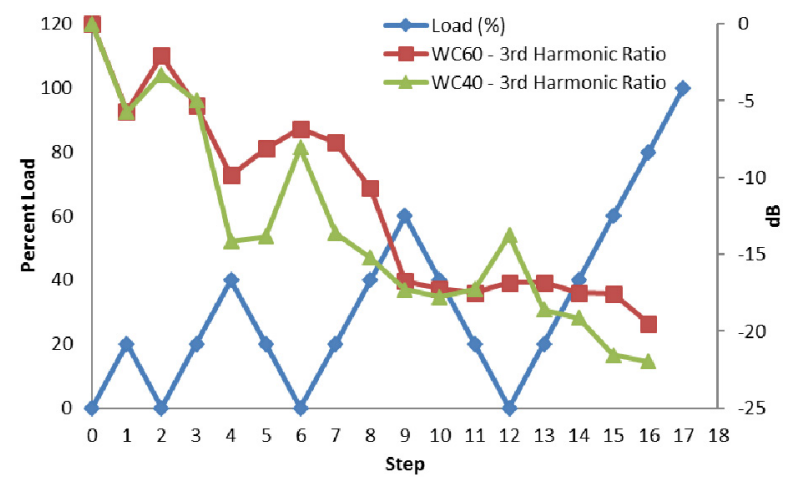

Fig. 10b Average 3rd harmonic amplitude with loading/unloading pattern.

consisting of aggregates. From studies (Solodov et al. 2011 ), the $3^{\text {rd }}$ harmonic amplitude was sensitive to contact friction. In $\mathrm{WC} 60$, there was less contact friction when repeated load was applied producing less sensitivity at the latter loading branches.

\subsection{Level of damage measurement for load- ing/unloading pattern}

In the loading/unloading pattern, two damage level measurements are introduced - total damage and the incremental damage. The total damage has a baseline of zero decibels when there is no stress experienced in the concrete at step load 0 as shown in Fig. 11. On the other hand, the incremental damage is the normalized harmonic ratio from zero load of any loading branch to the step load considered within the particular loading branch. The total damage of WC40 using normalized $3^{\text {rd }}$ harmonic ratio is $-22.37 \mathrm{~dB}$ from step load 0 to 16 , while the incremental damage at the last loading branch from step load 12 to 16 is $-8.23 \mathrm{~dB}$.

The $2^{\text {nd }}$ harmonic amplitude proved to be more consistent and sensitive than $3^{\text {rd }}$ harmonic amplitude for all water cement ratio when repeated load is applied. The total damage or difference of normalized $2^{\text {nd }}$ harmonic ratio from undamaged for WC40 and WC60 are -24.99 $\mathrm{dB}$ and $-22.37 \mathrm{~dB}$, respectively. Shown in Fig. 12 are the incremental damages of normalized $2^{\text {nd }}$ harmonic ratio considering only the last loading branch for WC40 and WC60 were $-10.88 \mathrm{~dB}$ and $-9.89 \mathrm{~dB}$, respectively. 


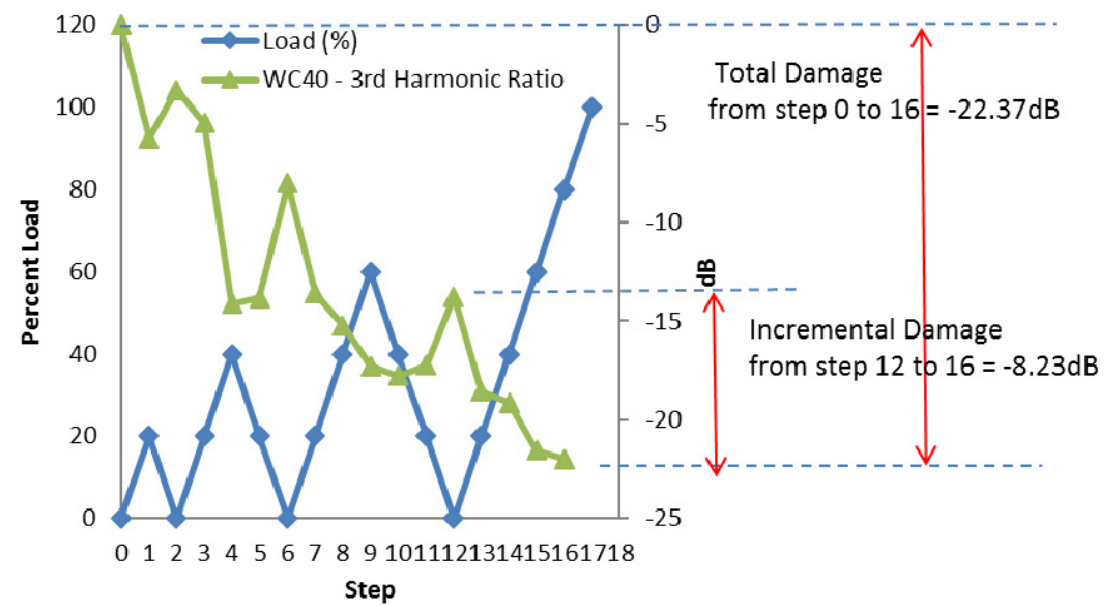

Fig. 11 Total damage and incremental damage for WC40 at the last loading branch.

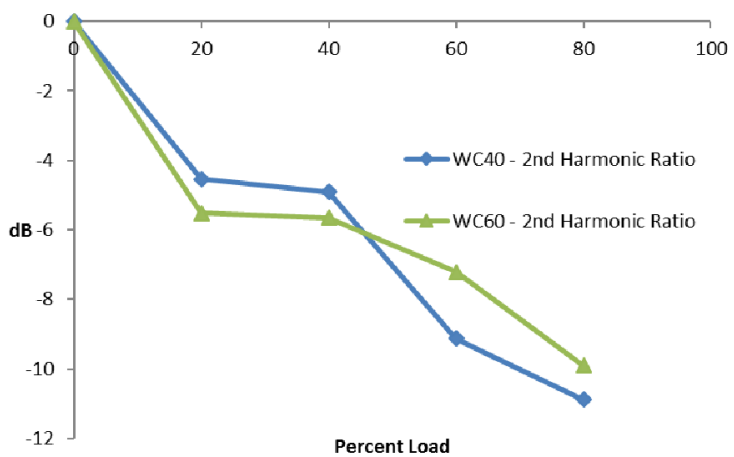

Fig. 12 Normalized 2nd and 3rd harmonic ratios for the incremental damage at the last loading branch.

\section{Conclusions}

From literatures, $3^{\text {rd }}$ harmonic amplitude was sensitive to internal damage. In this paper, it was investigated that the loading pattern played an important role in the behavior of nonlinear properties in strong and weak joints when subjected to load or repeated load. In reality, the concrete structure is assumed to be pre-loaded by its own weight or any initial loading condition subjected to it. Careful analysis of amplitude sensitivity was studied and the diminishing effect of harmonic generation was taken into consideration.

The experimental results showed that there was a good sensitivity for $3^{\text {rd }}$ harmonic amplitude $D_{\mathrm{A}}$ when it was subjected to single loading pattern for both WC40 and WC60. On the other hand, good sensitivity was observed for $2^{\text {nd }}$ harmonic amplitude $\mathrm{D}_{\mathrm{A}}$ when it was subjected to multiple loading/unloading pattern for both WC40 and WC60.

Furthermore, results showed that the single loading pattern produced $3^{\text {rd }}$ harmonic amplitude that was sensitive for both WC40 and WC60. The damage measurement using the largest difference of normalized $3^{\text {rd }}$ harmonic ratio from undamaged to damage state was $20.79 \mathrm{~dB}$ and $-14.79 \mathrm{~dB}$, respectively. On the other hand, the difference of normalized $2^{\text {nd }}$ harmonic ratio was $15.96 \mathrm{~dB}$ for WC40 and $-9.50 \mathrm{~dB}$ for WC60.

For the loading/unloading pattern, it produced differ- ent behavior on its harmonic generation. Second harmonic amplitude was used to measure the damage. The total damage or difference of normalized $2^{\text {nd }}$ harmonic ratio for WC40 and WC60 was $-24.99 \mathrm{~dB}$ and $-22.37 \mathrm{~dB}$, respectively. The incremental damage of normalized $2^{\text {nd }}$ harmonic ratio considering the last loading branch for WC40 and WC60 was $-10.88 \mathrm{~dB}$ and $-9.89 \mathrm{~dB}$ respectively.

\section{Acknowledgments}

This work has been supported by Japan Society for Promotion of Science (JSPS) under the Ronpaku Scholarship Program and KAKENHI Grand Numer 24246078.

\section{References}

Antonaci, P., Bruno, C. L. E., Gliozo, A., S. and Scalerandi, M., (2010). "Monitoring evolution of compressive damage in concrete with linear and nonlinear ultrasonic methods." Cement and Concrete Research, 40, 1106-1113.

Breysse, D., (2012). "Nondestructive evaluation of concrete strength: An historical review and a new perspective by combining NDT methods." Construction and Building Materials, 33, 139-163.

Daponte, P., Maceri, F. and Olivito, R. S., (1995). "Ultrasonic signal-processing techniques for the measurement of damage growth in structural materials." IEEE Transactions on instrumentation and measurement, 44(6), 1003-1008.

Hirose, S. and Achenbach, J. D., (1993). "Higher harmonics in the far field due to dynamic crack-face contacting." J. Acoust. Soc. Am., 93, 142-147.

Johnson, P. and Sutin, A., (2005). "Slow dynamics in diverse solids." J. Acoust. Soc Am., 117, 124-130.

Komlos, K., Popovics, S., Nurnbergerova, T., Babal, B. and Popovics, J. S., (1996). "Ultrasonic pulse velocity test of concrete properties as specified in various standards." Cement and Concrete Composites, 18, 357-364.

Liang, M. T. and Wu, J., (2002). "Theoretical elucidation on the empirical formulae for the ultrasonic testing method for concrete structures." 
Cement and Concrete Research, 32, 1763-1769.

Murase, M., Hayashi, T. and Kitayama, T., (2014). "Visualization of higher harmonic generation at contacting surfaces." JSNDI, 63, 310-315.

Shah, A. A., Ribakov, Y., (2009). "Non-linear ultrasonic evaluation of damaged concrete based on higher order harmonic generation." Materials and Design, 30, 4095-4102.

Shah, A. A., Ribakov, Y. and Hirose, S. (2009). "Nondestructive evaluation of damaged concrete using non-linear ultrasonics." Materials and Design, 30, 775-782.

Shah, A. A. and Ribakov, Y., (2008). "Non-linear nondestructive evaluation of concrete." Construction and Building Technology Journal, 2, 111-115.

Shah, A. A., Ribakov, Y. and Zhang, C., (2013). "Efficiency and sensitivity of linear and non-linear ultrasonics to identifying micro- and macro-scale defects in concrete." Materials and Design, 50, 905916.

Solodov, I. Y. and Chin, A. W., (1993). "Popping nonlinearity and chaos in vvibrations of contact interface between solids." Acoust. Phys., 39, 476-479.
Solodov, I. Y., Doring, D. and Busse, G., (2011). "New opportunities for NDT using non-linear interaction of elastic waves with defects." Journal of Mechanical Engineering, 57(3), 169-182.

Solodov, I. Y., Krohn, N. and Busse, G., (2002). "CAN: an example of nonclassical acoustic nonlinearity in solids." Ultrasonics, 40, 621-625.

Stauffer, D. J., Woodward, C. and White, K. R., (2005). "Non-linear ultrasonic testing with resonant and pulse velocity parameters for early damage in concrete." ACI Materials Journal, 102(2), 118- 121.

Oh, T., Kee, S. H., Arndt, R. W., Popovics, J. S. and Zhu, J., (2013). "Comparison of NDT methods for assessment of a concrete bridge deck." Journal of Engineering Mechanics, 139, 305-314.

Yim, H. J., Kim, J. H., Park, S. J. and Kwak, H. G., (2012). "Characterization of thermally damaged concrete using non-linear ultrasonic method." Cement and Concrete Research, 42, 1438-1446.

Zheng, Y., Maev, R. G. and Solodov, I. Y., (1999). "Nonlinear acoustic applications for material characterization: A review." Canadian Journal of Physics, 77, 927-967. 